SLAC-PUB-8459

hep-ex/0006012

May 2000

\title{
Status of PEP-II and BaBar *
}

\author{
J. Dorfan \\ Stanford Linear Accelerator Center, \\ Stanford University, Stanford, California 94309
}

Invited talk presented at

19th International Symposium on Lepton and Photon Interactions at High Energies Stanford University, Stanford, California

August 9-14, 1999

${ }^{*}$ Work supported by the Department of Energy, contract DE-AC03-76SF00515. 


\section{Introduction}

The SLAC B Factory asymmetric $B$-factories was approved by President Clinton in October 1993. B-factories The inaugural meeting of the detector collaboration was held at SLAC in December 1993, at which time the 9 nation BaBar collaboration began. First funding for the machine construction was released in January 1994, launching the SLAC/LBNL/LLNL PEP-II project collaboration [1]. The detector technical design report was completed in March 1995 [2].

PEP-II was completed in July 1998 and first collisions were observed on July 22, 1998. Commissioning without BaBar concluded in February 1999, at which time the BaBar detector commissioning ceased and BaBar was moved from the off-beamline to the on-beamline position. Running commenced in early May 1999, and first collisions with BaBar were observed on May 26, 1999.

In this report, the status of the PEP-II machine and the BaBar detector are presented. Performance will be presented as of the Lepton Photon Conference (August 1999) with subsequent performance levels footnoted as appropriate. This report is made on behalf of the SLAC/LBNL/LLNL team that built and commissioned PEPII, the SLAC Accelerator Department that commissioned and operate the machine, and the 71 institution, 9 nation detector collaboration that built, commissioned, and operate BaBar.

\section{PEP-II Machine}

The PEP-II PEP-II design parameters are shown in Table 1. The machine consists of two storage rings, vertically stacked - the high-energy (9 GeV) ring stores electrons and the low energy $(3 \mathrm{GeV})$ ring stores positrons (see Fig. 1). Collisions occur in a single interaction region (IR) (see Fig. 2), at which point the upper low energy ring (LER) has been brought down into the plane of the high energy ring (HER).

Collisions are head-on; beam separation is done magnetically. Electrons and positrons are supplied by the SLAC linac. Extraction and injection is done at the collision energy, so that no ramping of the stored beam is required. The RF system components are identical for the two rings-1.2 MW CW klystrons and warm copper cavities, which are actively damped. The on-cavity damping is not sufficient to ensure 


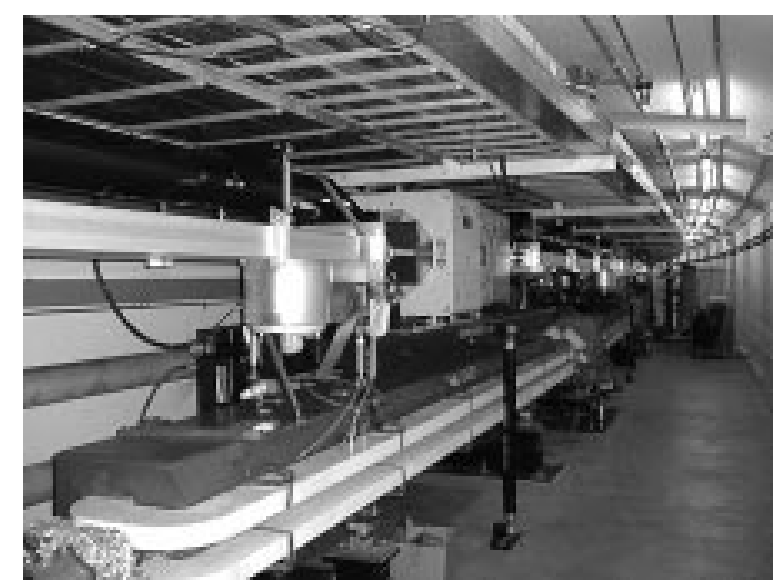

Figure 1: View of the two PEP-II storage rings.

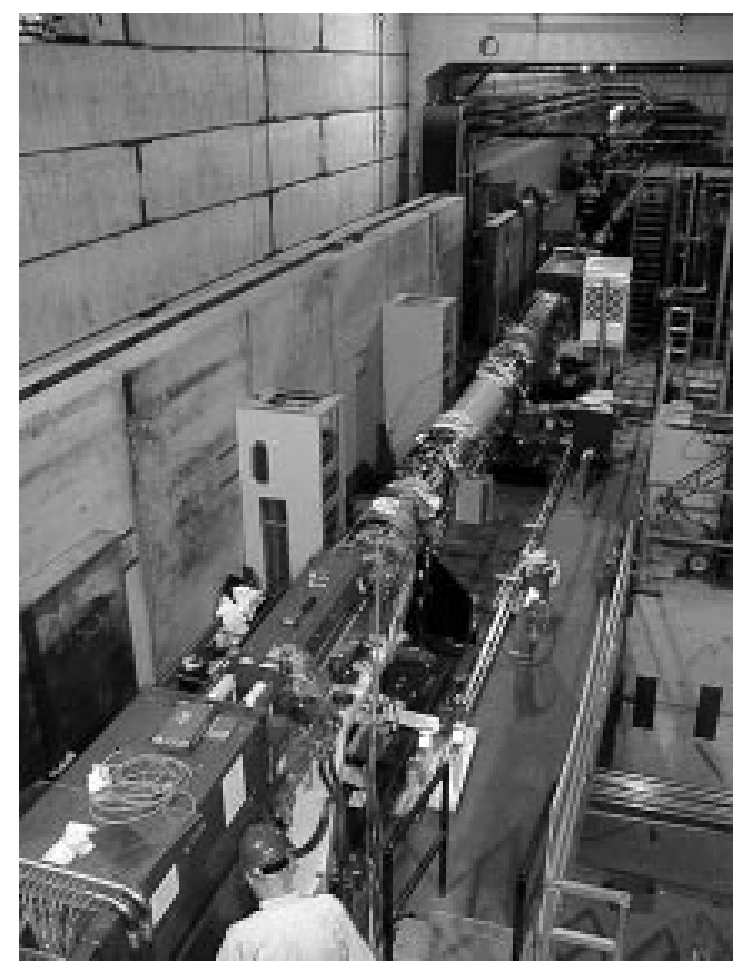

Figure 2: The PEP-II interaction region, before roll-in of the BaBar detector. 


\begin{tabular}{|c|c|c|}
\hline & $e^{+}$ & $e^{-}$ \\
\hline CM energy $(\mathrm{GeV})$ & \multicolumn{2}{|c|}{10.580} \\
\hline Beam energy $(\mathrm{GeV})$ & 3.119 & 8.973 \\
\hline Beam current (A) & 2.15 & 0.75 \\
\hline$\beta_{x}^{*} \mid \beta_{y}^{*}(\mathrm{~cm})$ & $50 \mid 1.5$ & $50 \mid 1.5$ \\
\hline \begin{tabular}{l|l}
$\epsilon_{x}$ & $\epsilon_{y}(\mathrm{~nm})$ \\
\end{tabular} & \begin{tabular}{l|l}
49 & 1.5 \\
\end{tabular} & \begin{tabular}{l|l}
49 & 1.5 \\
\end{tabular} \\
\hline$\sigma_{x}^{*}(\mu \mathrm{m})$ & \multicolumn{2}{|c|}{157} \\
\hline$\sigma_{y}^{*}(\mu \mathrm{m})$ & \multicolumn{2}{|c|}{4.7} \\
\hline$\sigma_{z}(\mathrm{~mm})$ & 12.3 & 11.5 \\
\hline Luminosity & \multicolumn{2}{|c|}{$3 \times 10^{33} \mathrm{~cm}^{-2} \mathrm{~s}^{-1}$} \\
\hline Tune shift & \multicolumn{2}{|c|}{0.03} \\
\hline Beam aspect ratio (v/h at IP) & \multicolumn{2}{|c|}{0.03} \\
\hline Number of colliding bunches & \multicolumn{2}{|c|}{1658} \\
\hline Bunch spacing $(\mathrm{m})$ & \multicolumn{2}{|c|}{1.26} \\
\hline Beam crossing angle & \multicolumn{2}{|c|}{0 (head-on) } \\
\hline
\end{tabular}

Table 1: PEP-II design parameters.

stable beams at high currents. Accordingly, bunch-by-bunch damping is supplied in both the transverse and longitudinal planes. The damping is primarily needed to ameliorate the effects of multi-bunch instabilities. A much more detailed description of PEP-II can be found in [1].

The machine group took on aggressive goals with respect to early peak and integrated luminosity performance. To achieve these goals, machine commissioning was phased in time. The high energy and low energy injection systems were completed early. The HER was completed in July 1997, allowing commissioning of the HER in 1997 and also testing of many systems that were common to the LER. The full machine was completed in July 1998 with the LER and IR being the last systems to finish. However, injection into a partial LER was achieved in early 1998. The phased approach to commissioning was an effective mechanism for early success with the full machine. Two weeks after completion of the full machine, the first collisions were observed. 


\section{BaBar Detector}

\begin{tabular}{|c|c|c|c|}
\hline Det. & Technology & Dimensions & Performance \\
\hline SVT & $\begin{array}{l}\text { Double-sided } \\
\text { silicon strips }\end{array}$ & $\begin{array}{l}5 \text { layers } \\
r=3.2 \text { to } 14.4 \mathrm{~cm} \\
-0.87<\cos \theta<0.96\end{array}$ & $\begin{aligned} \sigma_{z}= & \sigma_{x y}=50 \mu \mathrm{m} / p \\
& \oplus 15 \mu \mathrm{m} @ 90^{\circ} \\
\sigma_{\emptyset}= & \sigma_{\theta}=1.6 \mathrm{mr} / \mathrm{p} @ 90^{\circ}\end{aligned}$ \\
\hline $\mathrm{DC}$ & Drift chamber & $\begin{array}{l}40 \text { layers } \\
\mathrm{r}=22.5 \text { to } 80 \mathrm{~cm} \\
-111<\mathrm{z}<166 \mathrm{~cm}\end{array}$ & $\sigma_{p t} / p_{t}=\left[0.21 \%+0.14 \% \times p_{t}\right]$ \\
\hline PID & DIRC & $\begin{array}{l}1.75 \times 3.5 \mathrm{~cm}^{2} \text { quartz } \\
-0.84<\cos \theta<0.90\end{array}$ & $\begin{array}{l}N_{p e}=20-50 \\
\geq 4 \sigma K / \pi \text { separation for all } \\
\text { B decay products }\end{array}$ \\
\hline CAL & $\mathrm{CsI}(\mathrm{Tl})$ & $\begin{array}{l}16 \text { to } 17.5 X_{0} \\
\sim 4.8 \times 4.8 \mathrm{~cm} \text { crystals }\end{array}$ & $\begin{array}{l}\sigma_{E} / E=[1 \% / E(\mathrm{GeV})]^{1 / 4} \\
\quad \oplus 1.2 \% \\
\sigma_{\theta}=3 \mu \rho / \sqrt{E k(\mathrm{GeV})} \oplus 2 \mathrm{mr}\end{array}$ \\
\hline MAG & $\begin{array}{l}\text { Superconducting } \\
\text { segmented steel }\end{array}$ & $\begin{array}{l}\mathrm{IR}=1.40 \mathrm{~m} \\
\mathrm{~L}=3.85 \mathrm{~m}\end{array}$ & $\mathrm{~B}=1.5 \mathrm{~T}$ \\
\hline IFR & $\mathrm{RPC}$ & $\begin{array}{l}\text { 18-19 planar layers } \\
+4 \text { cylindrical layers }\end{array}$ & $\begin{array}{l}E_{\mu}>90 \% \\
\text { for } P_{\mu}>0.8 \mathrm{GeV} / \mathrm{c}\end{array}$ \\
\hline
\end{tabular}

Table 2: Performance specifications for the various components of the BaBar detector.

The BaBar BaBar detector design performance specifications are shown in Table 2. An isometric view of the detector is shown in Fig. 3, indicating the silicon vertex detector silicon vertex detector, drift chamber, Cherenkov radiation particle identification system, cesium iodide calorimeter, superconducting 1.5 Tesla magnet, and instrumented flux return [2]. The accelerator magnets in the IR come within 20 $\mathrm{cm}$ of the collision point. In fact, the vertex detector is actually mounted on the final accelerator magnets.

The vertex detector, one-half of which is shown in Fig. 4, is comprised of 5 layers of silicon strip detectors read out with radiation-hard electronics. The drift chamber 


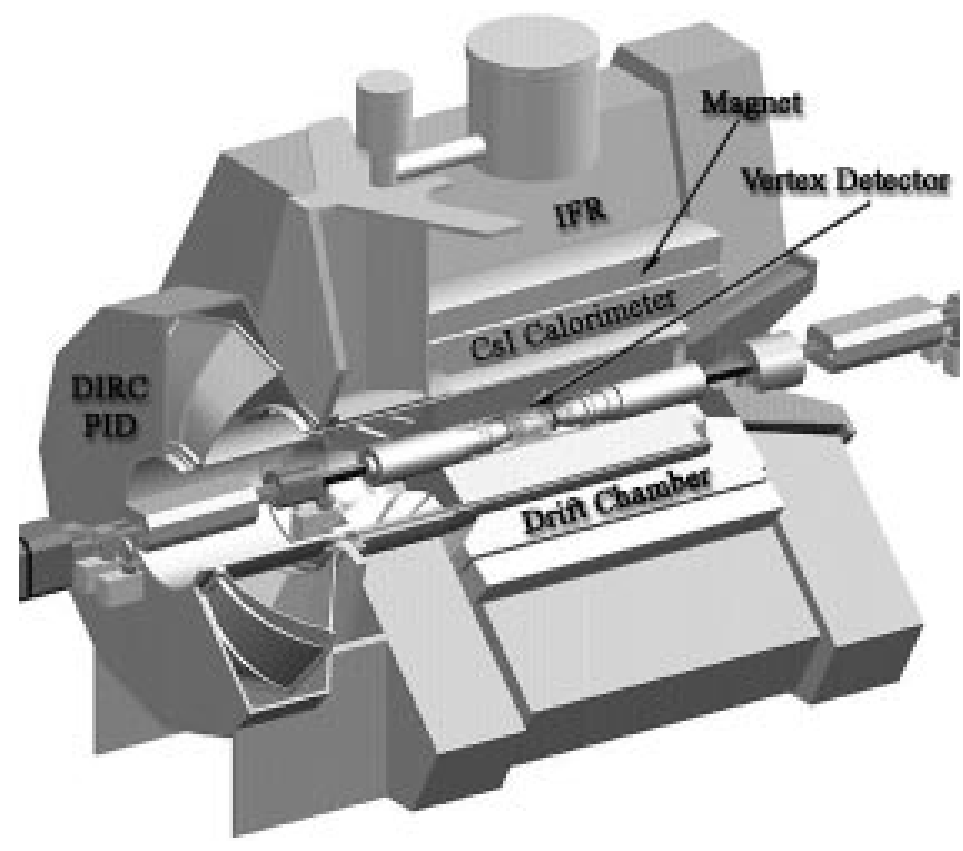

Figure 3: Cutaway view of the BaBar detector.

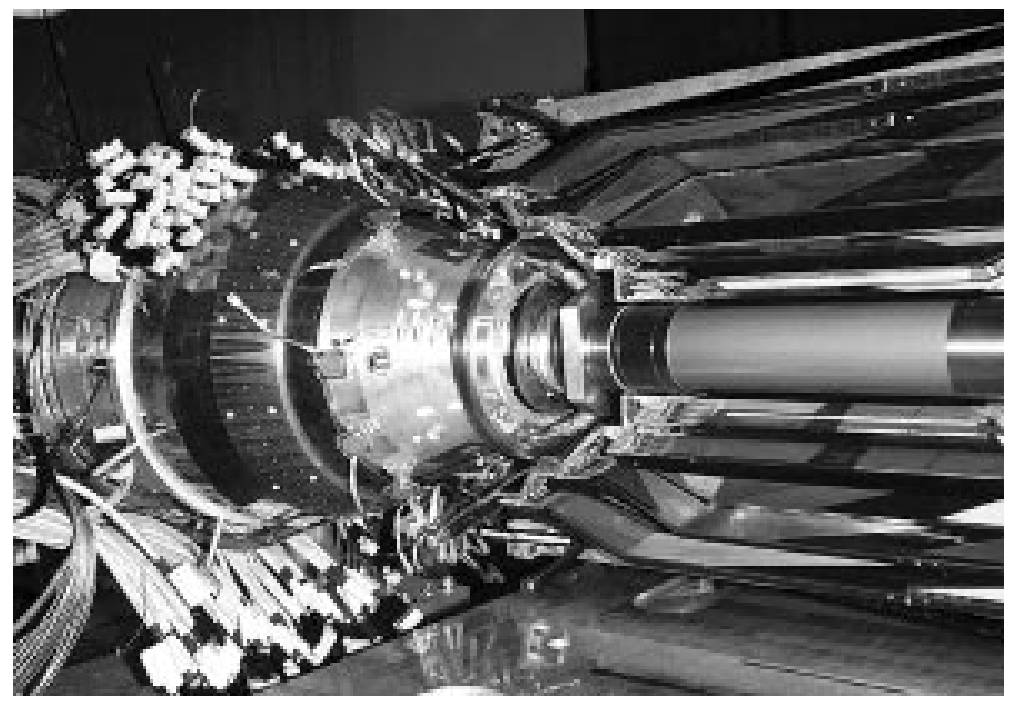

Figure 4: The BaBar silicon strip vertex detector. 


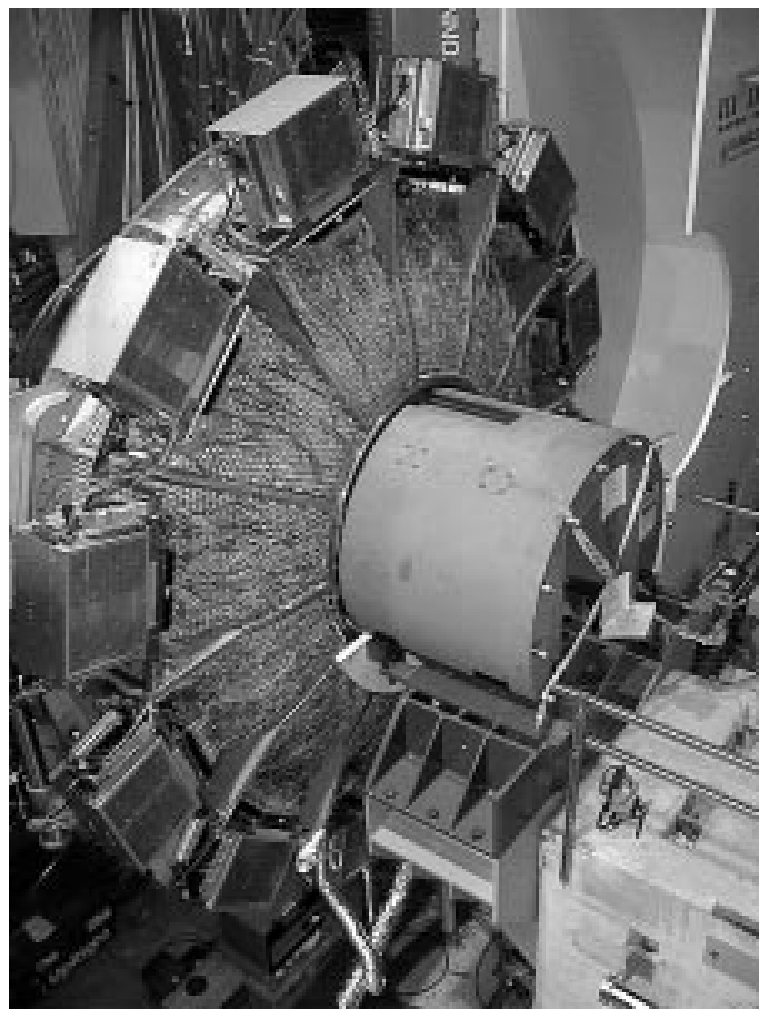

Figure 5: Phototube array for the BaBar DIRC particle identification system.

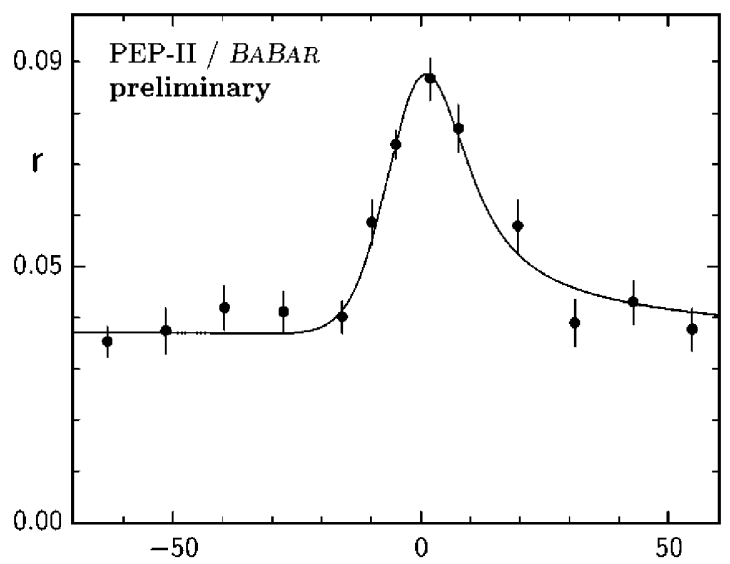

Figure 6: BaBar scan of the $\Upsilon(4 s)$ resonance. 
is a conventional, small cell design with 8 layers of axial wires interleaved with 4 layers of $\pm 3^{0}$ stereo wires. A low mass helium-based gas reduces multiple scattering. Surrounding the drift chamber is the DIRC Cherenkov detector system, 48 quartz bars running azimuthally. Cherenkov light generated in the quartz bars is propagated along the bars, exiting at the rear end of the detector where the exit angle is mapped by an array of 11,000 phototubes (see Fig. 5). The pattern of phototube hits can be used to measure the Cherenkov angle and thereby identify the radiating particle. Photons and electrons are identified in the cesium diodide calorimeter cesium iodide calorimeter. Because of the asymmetry of the machine, there is no need for electromagnetic calorimeter coverage in the backward region. The steel superstructure used to return the magnetic flux is made in layers interspersed with resistive plate chamers resistive plate chambers. This instrumented flux return is used for muon and $K_{L}^{0}$ identification. BaBar has chosen a pioneering approach to data acquisition and data analysis software. All software (both the coding and the databases) uses the object oriented approach The accelerator uses photons from the process $e^{+} e^{-} \rightarrow e^{+} e^{-} \gamma$ to derive a fast luminosity signal. However, the Bhabha rate is high enough at moderate luminosities to provide an online luminosity measurement from the detector. Luminosities quoted here come from the Bhabhas in BaBar.

\section{Machine and detector performance}

The month of June 1999 was used to integrate the machine and detector programs into a system which could run steadily for physics. The luminosity was improved and machine related backgrounds were understood. The Upsilon (4s) resonance $\Upsilon(4 s)$ resonance was mapped out in a 3 -day run as shown in Fig 6 . Using about $1 / 3$ of the data, resonance parameters of $M\left(\Upsilon_{4 s}\right)=10.5841 \pm 0.0007 \mathrm{GeV}$ and $\Gamma\left(\Upsilon_{4 s}\right)=11.1 \pm$ 3.4 MeV were obtained. In July 1999, persistent physics running began, interspersed with prolonged periods for machine development. Performance results for the machine as of the time of LP99 are given in Table 3. In the table, typical running parameters with BaBar are contrasted with the best that was achieved during commissioning without BaBar. As of the end of July 1999, BaBar had logged about $200 \mathrm{pb}^{-1}$, as 
can be seen from Fig. $7 .^{\dagger}$

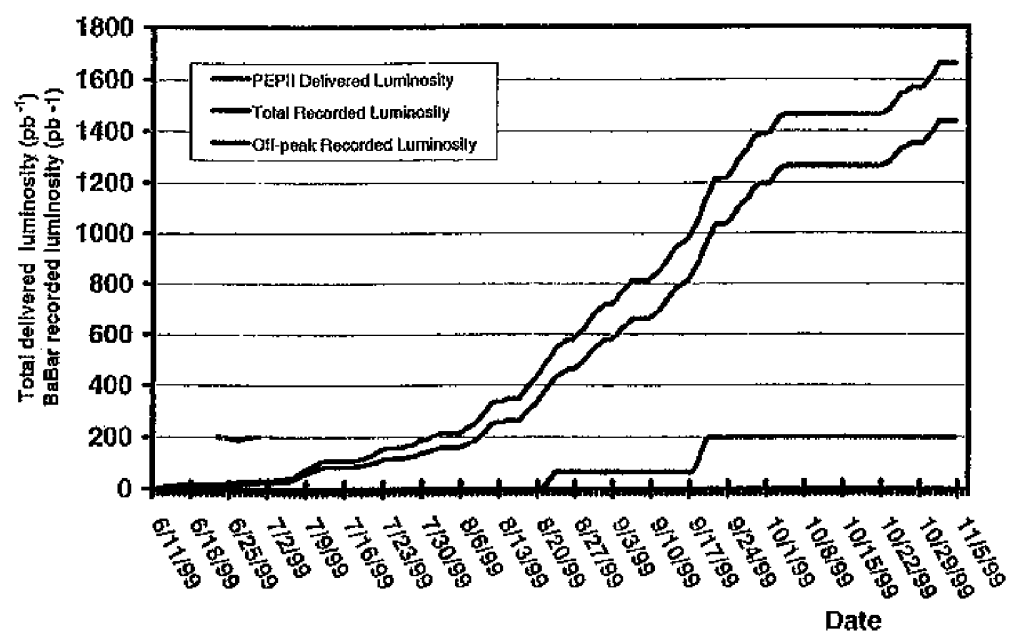

Figure 7: Integrated luminosity accumulated by PEP-II as a function of time.

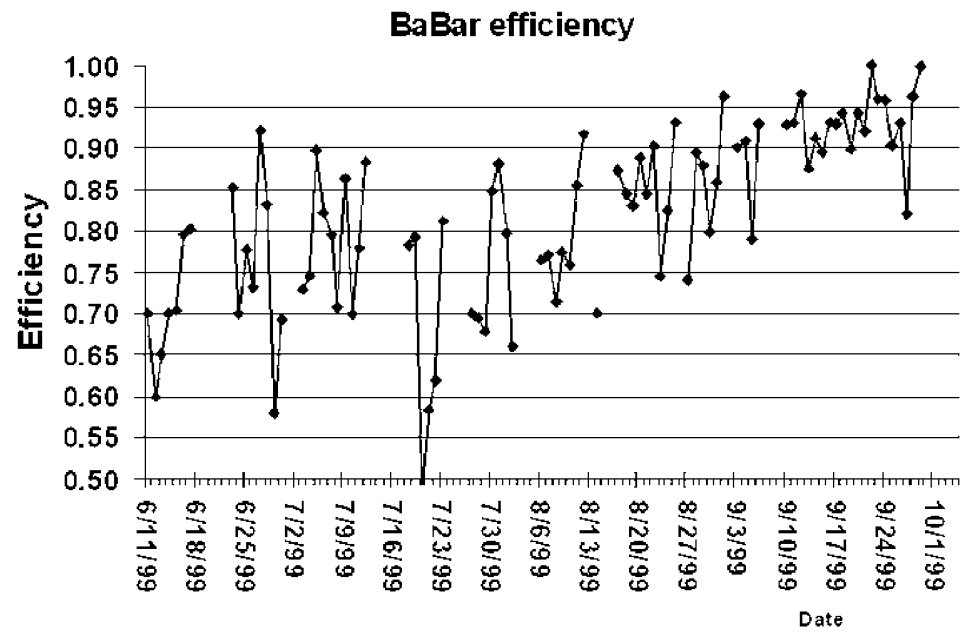

Figure 8: BaBar's efficiency of data logging, as a function of time.

Machine related backgrounds were reasonable; at luminosities above $5 \times 10^{32} \mathrm{~cm}^{-2}$ $\mathrm{sec}^{-1}$, all device occupancies were less than $20 \%$ of conservative allowances. Radiation dose levels were low; in the case of the highest radiation region of the Silicon Vertex Tracker (SVT), dose rates were $20 \%$ of the pre-ordained budget.

${ }^{\dagger}$ As of the end of November 1999, BaBar had logged $2 \mathrm{fb}^{-1}$, and the record peak luminosity was $1.43 \times 10^{+33} \mathrm{~cm}^{-2} \mathrm{sec}^{-1}$. 


\begin{tabular}{|c|c|c|c|c|}
\hline \multicolumn{5}{|c|}{ PEP-II HER Performance Results } \\
\hline Parameter & Units & Design & Commissioning & Running \\
\hline Energy & $\mathrm{GeV}$ & 9.0 & $\begin{array}{l}9.0, \text { ramp to } \\
9.1 \& \mathrm{bk}\end{array}$ & $\begin{array}{c}\text { 9.0, ramp to } \\
8.84-9.04\end{array}$ \\
\hline Single bunch current & $\mathrm{mA}$ & 0.6 & 12 & 0.55 \\
\hline Number of bunches & & 1658 & 1658 & 415 \\
\hline Total beam current & A & 0.995 & 0.75 & 0.25 \\
\hline Beam Lifetime & hours & 4 & $\begin{array}{c}8 \mathrm{hrs} \\
\text { @ } 250 \mathrm{~mA}\end{array}$ & $\begin{array}{c}6 \mathrm{hrs} @ \\
250 \mu \mathrm{amp}\end{array}$ \\
\hline Max. Injection Rate & $\mathrm{mA} / \mathrm{sec}$ & $2.1 @ 60 \mathrm{~Hz}$ & $2.5 @ 10 \mathrm{~Hz}$ & $0.6 @ 10 \mathrm{~Hz}$ \\
\hline \multicolumn{5}{|c|}{ PEP-II LER Performance Results } \\
\hline Energy & $\mathrm{GeV}$ & 3.1 & 3.1 & 3.1 \\
\hline Single bunch charge & $\mathrm{mA}$ & 1.3 & 7.0 & 1.9 \\
\hline Number of bunches & & 1658 & 1658 & 415 \\
\hline Total charge & A & 2.14 & 1.171 & 0.8 \\
\hline Beam Lifetime & hours & 4 & $\begin{array}{c}50 \mathrm{~min} \\
\text { @ } 800 \mathrm{~mA}\end{array}$ & $\begin{array}{c}120 \mathrm{~min} \\
\text { @ } 800 \mathrm{~mA}\end{array}$ \\
\hline Max. Injection Rate & $\mathrm{mA} / \mathrm{sec}$ & $5.9 @ 60 \mathrm{~Hz}$ & $3.0 @ 10 \mathrm{~Hz}$ & $2.7 @ 10 \mathrm{~Hz}$ \\
\hline \multicolumn{5}{|c|}{ PEP-II Collider Performance Results } \\
\hline Luminosity & $\mathrm{cm}^{-2} \mathrm{sec}^{-1}$ & $3 \times 10^{33}$ & $5.2 \times 10^{32}$ & $5.6 \times 10^{32}$ \\
\hline Specific Luminosity & $\mathrm{cm}^{-2} \mathrm{sec}^{-1} \mathrm{~mA}^{-2}$ & $3.1 \times 10^{30}$ & $1.7 \times 10^{30}$ & $2.1 \times 10^{30}$ \\
\hline Horizontal Spot Size & $\mu \mathrm{m}$ & 220 & 220 & 220 \\
\hline Vertical Spot Size & $\mu \mathrm{m}$ & 6.6 & 8.6 & 10.8 \\
\hline
\end{tabular}

Table 3: Performance of PEP-II. "Commissioning" refers to the best results obtained without the detector in place. "Running" refers to typical results with BaBar. 
As can be seen from Fig. 8, the detector data logging efficiency is high, particularly at this early phase of operation. Typically, BaBar logs $\geq 90 \%$ of the delivered luminosity. It is much too early to expect physics results. However, some plots are shown which are representative of the process of tuning up and monitoring the performance of BaBar. This is an evolving process and much work remains to be done.

Figure 9 shows early performance measures for the SVT. If one excludes 8 nonworking modules (out of 208), detector efficiencies are seen to be excellent. The hit resolution in $\phi$ and $z$ are not far from that expected.

Figure 10 shows the drift chamber single-hit resolution as a function of distance from the wire. This resolution is very close to the design performance of $140 \mu$ averaged over the cell. The current status of the $d E / d x$ performance is also shown in Fig. 10. The two dark spots at high momentum are the Bhabha electrons and positrons.

Figure 11 shows the $\gamma \gamma$ mass spectrum for di-gamma energies above $500 \mathrm{MeV}$. A clear $\pi^{0}$ peak is visible - the resolution on the $\pi^{0}$ is about $30 \%$ larger than design.

Figure 12 shows the effect of Cherenkov detector particle identification using the DIRC system. The $K^{ \pm} \pi^{\mp}$ mass spectrum in the region of the $D^{0}$ is shown without using particle identification and using the DIRC to identify the kaon.

Figure 13 shows the $D^{*}-D^{0}$ mass difference, using the two $D^{0}$ decay modes, $K^{ \pm} \pi^{\mp}$ and $K^{ \pm} \pi^{\mp} \pi^{0}$ (no particle ID is used). These plots are indicative of good early performance of the tracking systems.

Figure 14 shows the di-lepton invariant mass spectra in the region of the $J / \psi$ particle.

While it is much too early to expect high quality (design level) performance from the detector, these plots demonstrate rather impressive performance this early in the life of the detector.

\section{$5 \quad$ Future Plans}

As of LP99, the accelerator was complete, but BaBar was missing $2 / 3$ of the quartz bars. These bars were installed in October 1999, thereby completing the detector. The facility will run until Summer 2000, with a short break in December 1999. 
BaBar SVT hit resolution
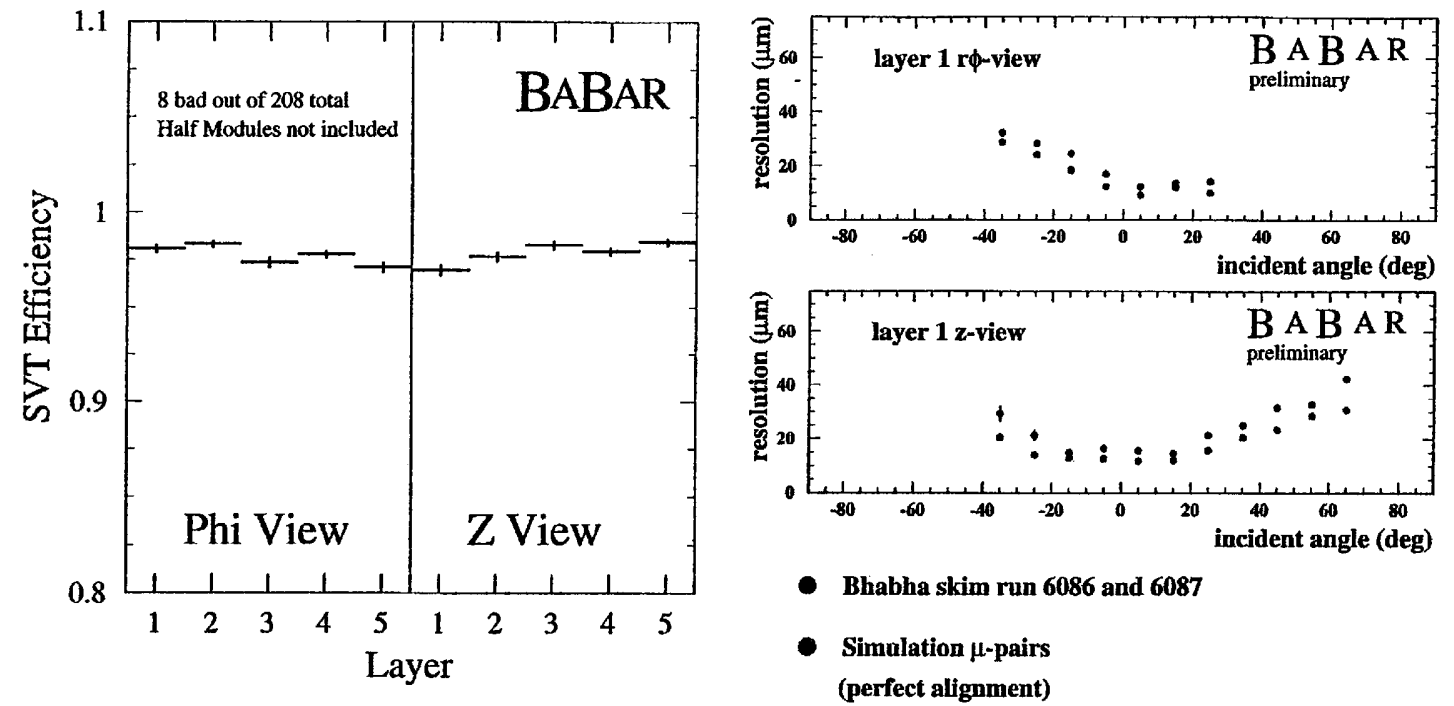

Figure 9: Performance of the BaBar silicon vertex detector. Left: efficiency; Right: tracking resolution.
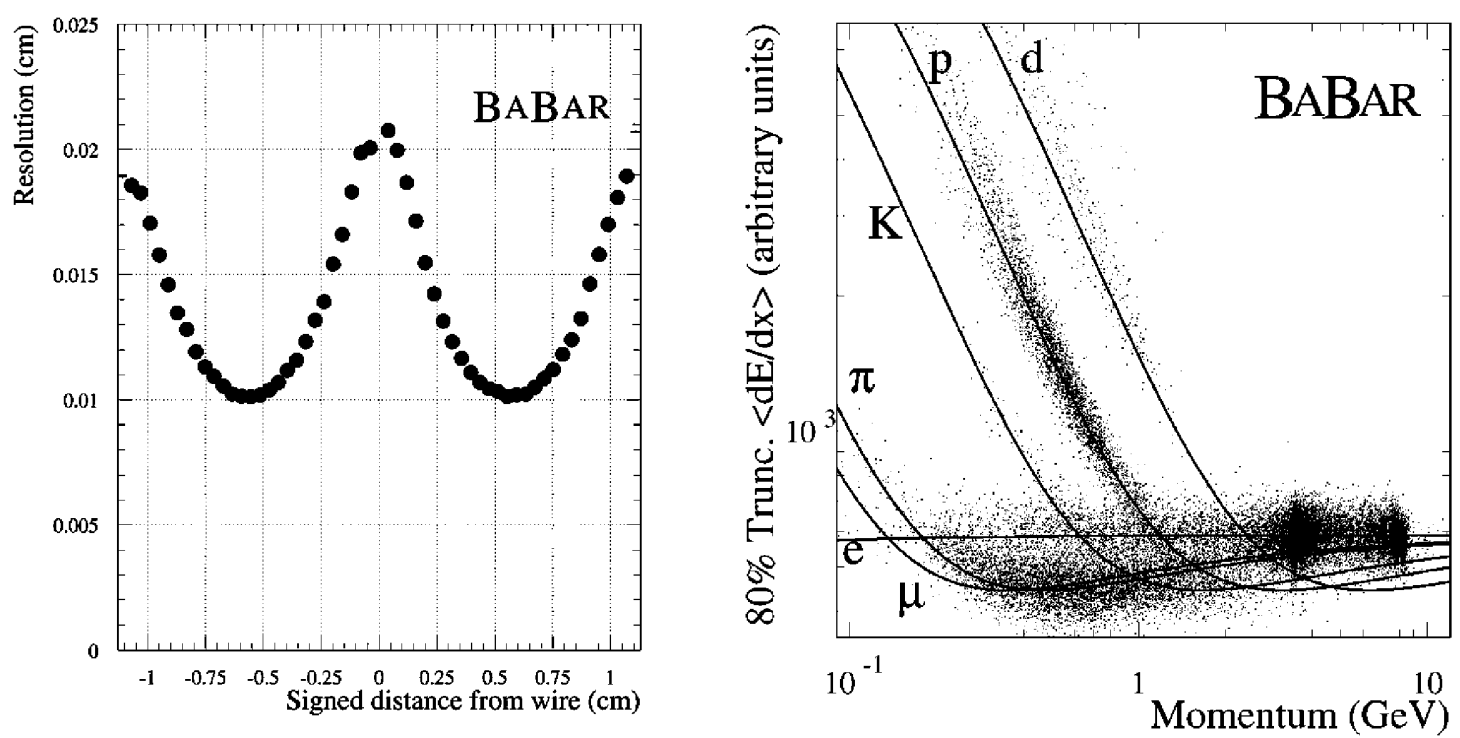

Figure 10: Performance of the BaBar drift chamber. Left: tracking resolution; Right: $d E / d x$. 


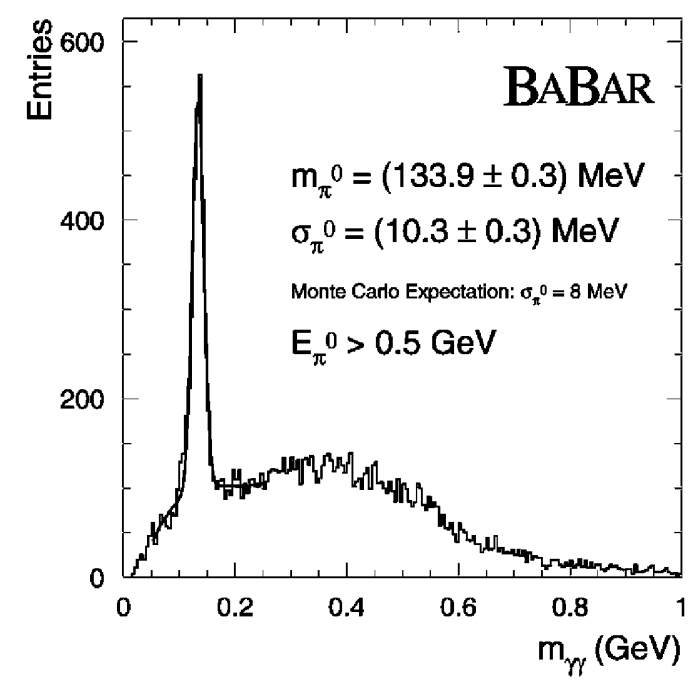

Figure 11: Two-photon mass spectrum observed by the BaBar detector.
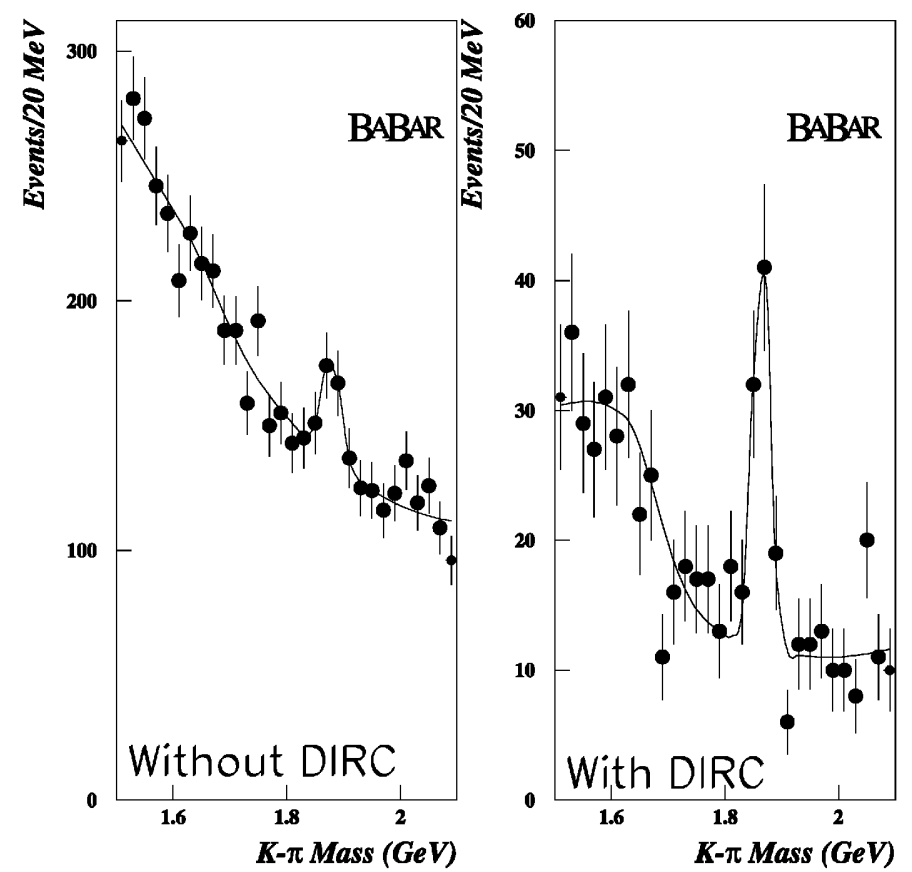

Figure 12: $K^{ \pm} \pi^{\mp}$ mass spectrum observed by the BaBar detector. Left: with no particle ID; Right: including the DIRC system. 

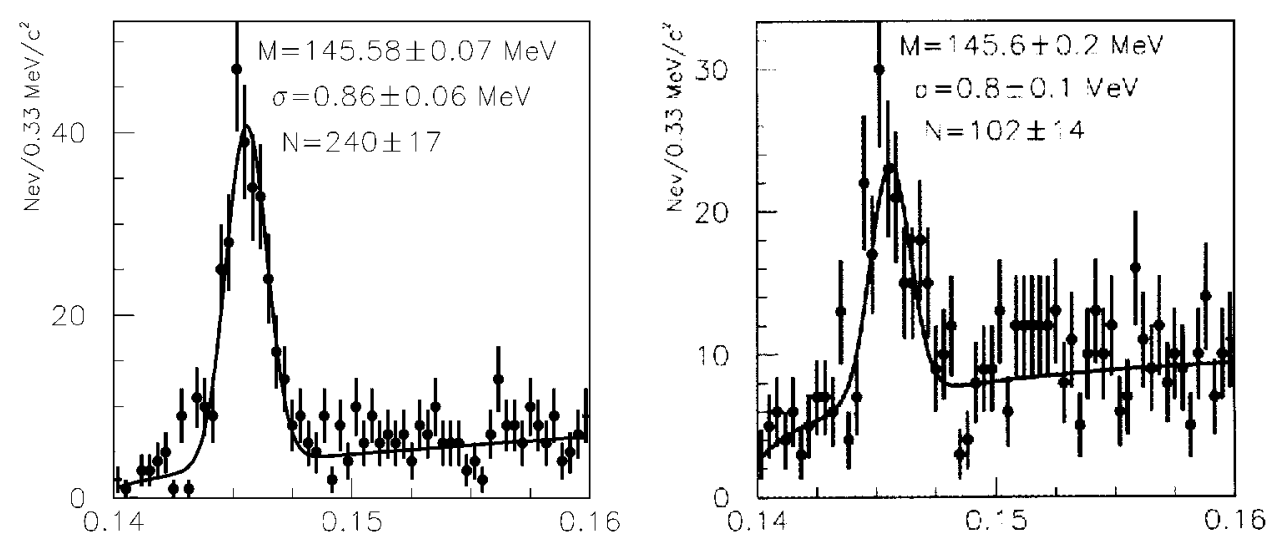

Figure 13: $D^{*}-D^{0}$ mass difference, as reconstructed by the BaBar detector. Left: $D^{0} \rightarrow K^{ \pm} \pi^{\mp}$; Right: $D^{0} \rightarrow K^{ \pm} \pi^{\mp} \pi^{0}$.

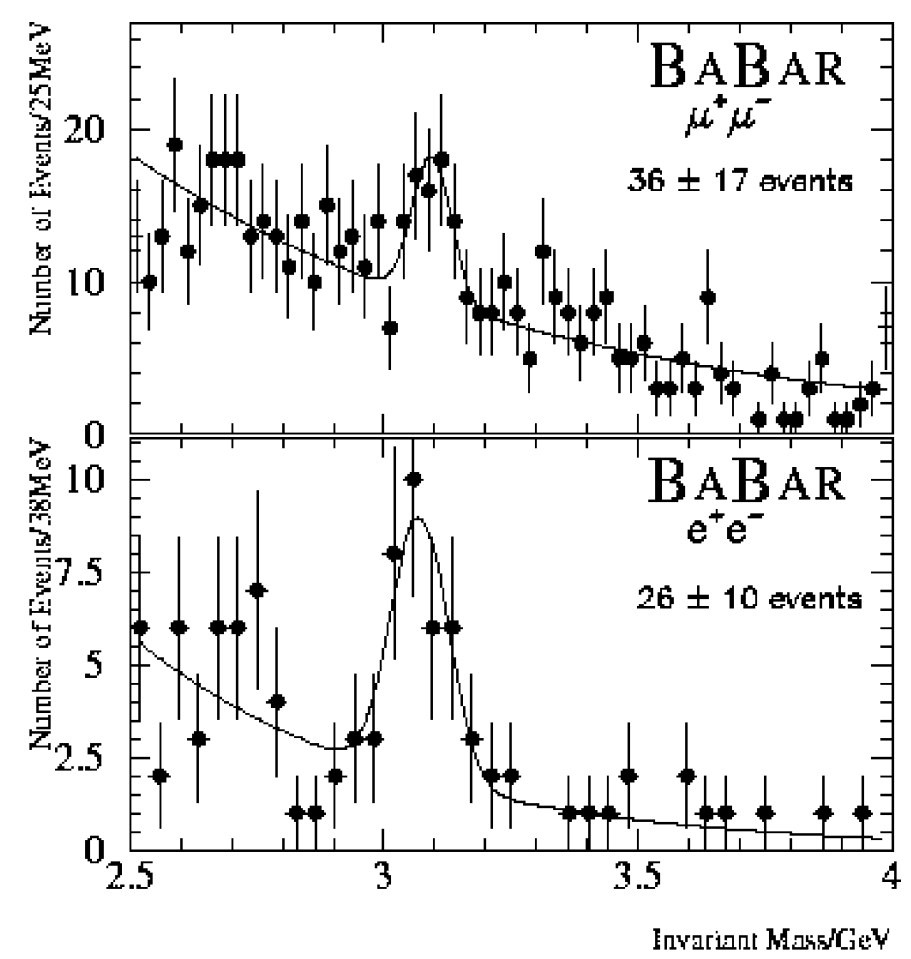

Figure 14: Dilepton invariant mass spectrum in BaBar events, showing the $J / \psi \rightarrow$ $\ell^{+} \ell^{-}$peak. 


\section{References}

[1] PEP-II, an Asymmtric B Factory: A Conceptual Design Report, LBL-PUB-5379, SLAC-R-418, CALT-68-1869, UCRL-ID-114055, UC-IIRPA-93-01 (1993).

[2] D. Boutigny et al. [BaBar Collaboration] BaBar Technical Design Report, SLACR-0457 (1995). 